\title{
Emergency Preparedness in Dietetics during a Pandemic: Lessons Learned from an ICU Dietitian during Covid-19
}

Pamela Rothpletz-Puglia

Rutgers University, pr.puglia@shp.rutgers.edu

Michelle D. Mena

Rutgers University, michelledmena@gmail.com

Follow this and additional works at: https://nsuworks.nova.edu/ijahsp

Part of the Dietetics and Clinical Nutrition Commons

\section{Recommended Citation}

Rothpletz-Puglia P, Mena MD. Emergency Preparedness in Dietetics during a Pandemic: Lessons Learned from an ICU Dietitian during Covid-19. The Internet Journal of Allied Health Sciences and Practice. 2021 Jan 01;19(3), Article 12.

This Concept Piece is brought to you for free and open access by the College of Health Care Sciences at NSUWorks. It has been accepted for inclusion in Internet Journal of Allied Health Sciences and Practice by an authorized editor of NSUWorks. For more information, please contact nsuworks@nova.edu. 


\title{
Emergency Preparedness in Dietetics during a Pandemic: Lessons Learned from an ICU Dietitian during Covid-19
}

\begin{abstract}
Purpose: The incidence, prevalence, and chaos of the Covid-19 disease sequelae is an adverse event akin to a natural disaster or wartime creating a high degree of uncertainty and vulnerability for health care providers. This is an account of a Registered Dietitian Nutritionist's (RDN) experience on the frontlines during an epidemic providing care to patients with Covid-19. The purpose of the series of discussions was to create a live account of a frontline RDN's experience during the pandemic to develop recommendations for emergency preparedness in dietetics during Covid-19. Methods: Since the Covid-19 pandemic is causing significant hardship, loss of life, and changes in health care provider roles, particularly in the intensive care unit (ICU), we conducted a real-time job analysis of an ICU RDN in New York during the height of the pandemic in that region. Critical Incident Technique (CIT) has been gradually refined and is used for curriculum development, performance evaluation, and for the creation of professional standards in health professions jobs. To gain an in-depth understanding of the job of an ICU RDN on the frontlines during Covid-19, CIT was used an information-gathering strategy in a series of 7, hour-long meetings via Zoom, an online meeting platform. These meetings occurred during the apex of the Covid-19 pandemic in NYC from March 2020 - June 2020. Results: Covid-19 resulted in RDN decision-making that involved circumstances with a high degree of variability and uncertainty. There were several mediators that enabled the RDN to be resilient and adapt to the adversity of Covid-19 in this hospital. Conclusions: The RDN's account of her experience during Covid-19 resulted in several recommendations for ICU emergency preparedness. This account also elucidates the trauma and resilience experienced by a health care provider during Covid-19.
\end{abstract}

\section{Author Bio(s)}

Pamela Rothpletz-Puglia, EdD, RDN is the Director of the Master of Science in Health Professions Education program and an Associate Professor at Rutgers, The State University of New Jersey. She is also a Research Advisor for the Department of Interdisciplinary Studies and a member of the Methodology and Statistical Support Team.

Michelle D. Mena, MS, RDN, CNSC is a Registered Dietitian currently employed as a Critical Care Dietitian for the Medical Intensive Care Unit at a hospital in Long Island, NY.

\section{Acknowledgements}

Michelle Mena: I would like to thank my RDN and DTR colleagues for all their hard work during the pandemic and for being part of the inspiration for this article. 


\title{
IUAHSP \\ The Internet Journal of Allied Health Sciences and Practice \\ Vol. 19 No. 3 ISSN 1540-580X
}

\section{Emergency Preparedness in Dietetics during a Pandemic: Lessons Learned from an ICU Dietitian during Covid-19}

\author{
Pamela Rothpletz-Puglia \\ Michelle D. Mena \\ Rutgers University
}

United States

\begin{abstract}
Purpose: The incidence, prevalence, and chaos of the Covid-19 disease sequelae is an adverse event akin to a natural disaster or wartime creating a high degree of uncertainty and vulnerability for health care providers. This is an account of a Registered Dietitian Nutritionist's (RDN) experience on the frontlines during an epidemic providing care to patients with Covid-19. The purpose of the series of discussions was to create a live account of a frontline RDN's experience during the pandemic to develop recommendations for emergency preparedness in dietetics during Covid-19. Methods: Since the Covid-19 pandemic is causing significant hardship, loss of life, and changes in health care provider roles, particularly in the intensive care unit (ICU), we conducted a real-time job analysis of an ICU RDN in New York during the height of the pandemic in that region. Critical Incident Technique (CIT) has been gradually refined and is used for curriculum development, performance evaluation, and for the creation of professional standards in health professions jobs. To gain an in-depth understanding of the job of an ICU RDN on the frontlines during Covid-19, CIT was used an information-gathering strategy in a series of 7, hour-long meetings via Zoom, an online meeting platform. These meetings occurred during the apex of the Covid-19 pandemic in NYC from March 2020 - June 2020. Results: Covid-19 resulted in RDN decision-making that involved circumstances with a high degree of variability and uncertainty. There were several mediators that enabled the RDN to be resilient and adapt to the adversity of Covid-19 in this hospital. Conclusions: The RDN's account of her experience during Covid-19 resulted in several recommendations for ICU emergency preparedness. This account also elucidates the trauma and resilience experienced by a health care provider during Covid- 19 .
\end{abstract}

Keywords: emergency preparedness, dietetics, ICU, pandemic, critical incident technique 


\section{INTRODUCTION}

The incidence, prevalence, and chaos of the Covid-19 disease sequalae is an adverse event akin to a natural disaster or wartime creating a high degree of uncertainty and vulnerability for health care providers. Guidance on both critical care nutrition and malnutrition for Covid-19 has been published, and there are a number of publications about the trauma experienced by health care providers during Covid-19.1-8 This is an account of a Registered Dietitian Nutritionist's (RDN) experience on the frontlines during an epidemic providing care to patients with Covid-19.

Since the Covid-19 pandemic is causing significant hardship, loss of life, and changes in health care provider roles, particularly in the intensive care unit (ICU), we conducted a real-time job analysis of an ICU RDN in New York during the height of the pandemic in that region. In organizational psychology, a job analysis is the process of obtaining information about how jobs are performed.., 10 The critical incident technique (CIT) emerged as a job analysis strategy during World War II to scientifically categorize human behaviors to rapidly reduce pilot error during the war. ${ }^{11} \mathrm{CIT}$ has been gradually refined and is used for curriculum development, performance evaluation, and for the creation of professional standards in health professions jobs. ${ }^{12,13}$

To gain an in-depth understanding about the job of an ICU RDN on the frontlines during Covid-19, CIT was used an information gathering strategy in a series of 7 , hour long meetings via Zoom, an online meeting platform. These meetings occurred during the apex of the Covid-19 pandemic in NYC from March 2020 - June 2020. The information gathered during the meetings included discussions about the situation and problem, the actions taken including problem solving and rapid improvisations in clinical care due to the circumstances, outcomes, and recommendations. The purpose of the series of discussions was to create a live account of a frontline RDN's experience during the pandemic to develop recommendations for emergency preparedness in dietetics during Covid-19.

\section{SITUATION}

To provide context, this story is about an RDN certified in nutrition support with close to fifteen years of experience, working in a 20-bed medical ICU at a hospital in suburban New York. The first case of Covid-19 to arrive at the RDN's hospital was on March 5,2020 , a date that the RDN recalled vividly and repeated in several of the meetings. The hospital all around had been preparing by discharging patients accordingly, while the ICU increased its negative pressure rooms to full capacity. ICU beds, both medical and surgical, were filling up quickly with both confirmed and suspect Covid-19 cases. Because it took time for the test results to return, this time was described by the RDN as "the calm before the storm."

The maximum number of ventilated patients in this RDN's ICU on any given day pre-Covid-19 was around ten, or half of the ICU. By the end of March 2020, all intensive care units (medical, surgical and neuro) were at maximum capacity with ventilated patients - 54 beds total-which could overwhelm any healthcare worker. "I never in my life had 20 ventilated patients to care for at one time." By mid-April, that number had increased to around 140 ventilated patients. The post-anesthesia care unit (PACU), general conference room, cardiac catheter lab and many other spaces were converted to house ICU patients to accommodate this tremendous increase (267\%) that became the new Covid-19 reality.

The extensive media coverage at the time reported on the lack of Covid-19 testing, personal protective equipment (PPE), and ventilators. However, unbeknownst to the media or general public, the RDN's institution was also faced with the dilemma of enteral feeding pumps and gravity feeding bags also being in short supply. The Covid-19 disease sequalae involved complications that "wreaked havoc" on the body such as blood clotting issues and acute respiratory distress syndrome which required patients to lie in the prone position for 16 hours per day. In addition, the length of stay among ventilated patients with Covid-19 compounded the feeding pump and feeding schedule problem as patients remained on ventilators for significant amounts of time. The lack of these supplies necessitated the RDNs to create protocols for feeding schedules to allow for "pump sharing" and bolus feeding, along with the emergent ordering of gravity feeding bags. Communication was instrumental among the hospital RDNs. Steps such as daily communication/updates and protocol development were implemented to reinforce a team-based approach to care being employed by the RDNs to ensure consistent nutritional interventions were being implemented during this pandemic.

The morbidity and mortality of Covid-19 challenged health care providers in numerous ways as the RDN explained, "Some patients don't exactly do worse, but they're not doing better either...they're just kind of in limbo, essentially. One person who was able to come off the vent after 18 days was on continuous renal replacement therapy. She was one patient that was able to be transitioned to dialysis but because of Covid-19 she will need dialysis for the rest of her life." The RDN also recalled the death rate being significantly higher than normal in the ICU during the peak of the pandemic, stating, "I lost almost an entire ICU's worth of patients

(C) The Internet Journal of Allied Health Sciences and Practice, 2021 
in less than two weeks. Like I said, I'm used to death. I've worked in hospitals for many years. I'm used to it. Death does not get to me, but I think the amount of dead bodies that l've seen is just -."

\section{ACTIONS}

Covid-19 resulted in RDN decision making that involved circumstances with a high degree of variability and uncertainty. To help cover the influx of ventilated patients, RDNs who normally provide care in other units had to quickly transition their scope of practice in order to provide care for these critically-ill, ventilated patients. The RDN commented "My colleagues - all of a sudden everyone became a critical care dietitian...this has been a huge strain for them because most dietitians are working in their own niche, and now they are covering ventilated patients in ICU settings."

In addition to the strain placed on RDNs to practice in areas unfamiliar to them, Covid-19 presented new challenges to seasoned ICU RDNs. Before Covid-19 it was uncommon in the ICUs for so many patients at once to require proning/supining. "We didn't have any guidelines exactly in place for feeding while in the prone position." Initially patients were not being fed optimally, but the RDNs rectified the situation by creating enteral nutrition feeding protocols for dissemination and implementation, which in turn led to the development of a standardized feeding protocol for ventilated patients in the prone position as part of the policies and procedures within their hospital system. Recommendations included enteral nutrition being given at a trophic rate, holding enteral feeds for up to an hour prior to proning or suping, ensuring the head of the bed (HOB) is greater than 10-25 degrees, the use of IV erythromycin ( $250 \mathrm{mg}$ every $6 \mathrm{hrs}$ ) to assist with management of volume tolerance and reduce the incidence of vomiting, and the option for the placement of a post pyloric tube if necessary.

Too few feeding pumps and gravity bags also led to daily inventory being taken of these supplies, and subsequent decisions being made to overcome these resource limitations to best meet the nutritional needs of each patient. Enteral feeding pumps were ultimately shared in the medical ICU, while other make-shift ICU units utilized bolus feeding and/or gravity bag feeding regimens Signs were posted outside patient rooms in the medical ICU indicating the timing of the feeding regimen along with information regarding how the feeding pumps were being utilized.

The RDN also explained that Covid-19 had implications for the way she communicated with families. Since families could not be with their loved ones during Covid-19, healthcare providers communicated with families by telephone instead of at the bedside. This change led to less RDN communication with families of ventilated patients. This was done purposefully as to not overwhelm families given what their loved ones were going through, and their inability to visit them. Many times, the RDN also described the difficulty and sympathy for patients suffering alone due to Covid-19. In one discussion she described how a healthcare provider held a phone with a pastor on the line before the patient died.

\section{OUTCOMES}

There were several mediators that enabled the RDN to be resilient and adapt to the adversity of Covid-19 in this hospital. Since Covid-19 overwhelmed the entire institution, institutional and leadership flexibility were described as integral to the RDN's ability to make decisions and provide quality care. The clinical leadership at this hospital remained in constant communication with other hospitals in its health system. Since there were numerous patients on ventilators, these institutions readjusted their nutrition assessment and follow-up policies to allow time for the RDNs to prioritize these patients. Other institutional and leadership measures taken included the allowance of RDNs to adjust work schedules such as coming to work on a weekend day in order to take off a day during the week. This flexibility enabled the RDN to take a break and practice self-care during these trying times.

The RDN also spoke in relation to the need for self-care such as sleep, connecting with family/friends, and time off. This RDN exhibited a high degree of positive reappraisal, a positive coping skill defined as the ability to review the negative event in a positive light, also known as reframing. ${ }^{14,15}$ For example, "Everyone is on a learning curve. We're all, every discipline trust me, will learn something from this and will in some way have to adapt our practice or adapt our guidelines when it comes to this specific virus and other pandemics." The RDN attributed her coping to self-care, but also acknowledged that acts of humanity such as community-members providing meals to healthcare workers were inspiring. The RDN also discussed how teamwork among the RDNs supported the work and increased morale and commitment.

The trauma experienced by health care providers during Covid-19 has been described, and the RDN's experiences underscore this outcome. 3-5,7,8 The RDN stated, "It just was so overwhelming, I came home, I kind of just like checked out mentally. I didn't really want to think about what was going on, but at the same time I couldn't stop." Preserving the mental health and wellbeing of health care providers and other staff came up in numerous discussions. 


\section{RECOMMENDATIONS FOR EMERGENCY PREPAREDNESS IN DIETETICS}

The RDN's account of her experience during Covid-19 resulted in several recommendations that fell into the following categories and are summarized in the Table.

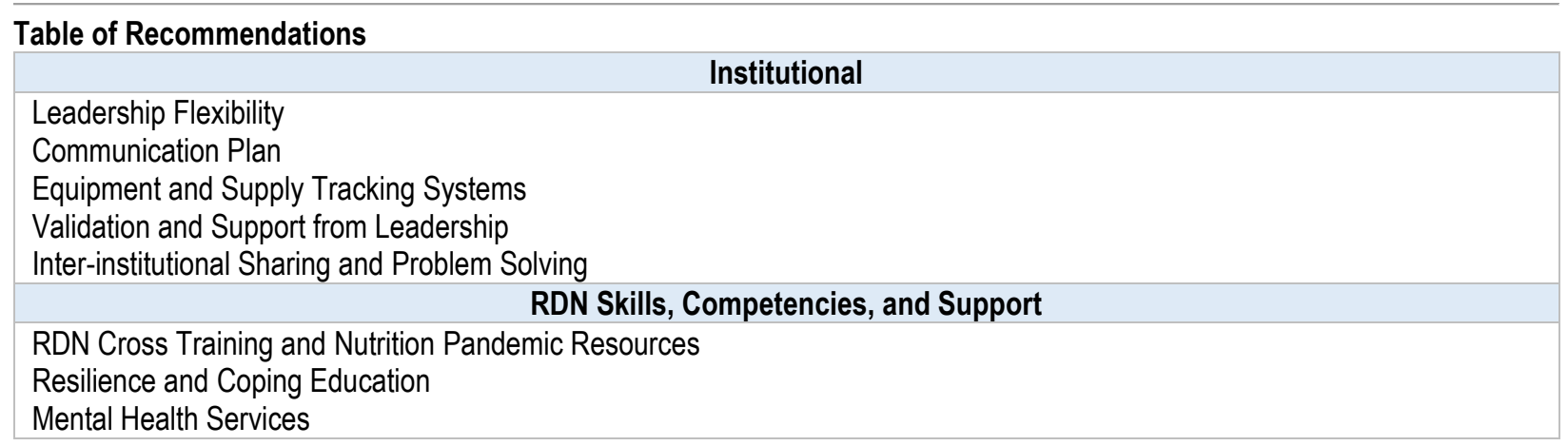

\section{Institutional}

Regardless of institution size or location, healthcare leadership should strive to support the well-being of those involved in patient care. ${ }^{16}$ Crisis leadership strategies should include flexibility, listening, remaining calm, communication, demonstrating responsibility, acknowledging the gravity of the situation, debriefing and updating staff regularly, and providing regular praise for the RDNs navigating unprecedented and exceptional circumstances. ${ }^{7}$

\section{Communication Plan}

Having a communication board accessible to staff enables communication and allows for the rapid barrage of issues in a pandemic to be addressed quickly. Messages should be clear and provide the best practices in order to address clinicians' concerns and expectations during a pandemic. ${ }^{16} \mathrm{~A}$ communication plan is integral to future guidelines for emergency preparedness within departments. However, it is important to keep in mind that "information overload", or an influx of communications via specific channels such as multiple emails, could have a negative impact on a healthcare worker's ability to respond to messages in an efficient and appropriate manner during an emergency. ${ }^{17}$ Verbal review of any important changes could be a crucial aspect of communication for emergency preparedness.

\section{Equipment and Supply Tracking Systems}

Implementing a tracking system to account for equipment in the hospital can be essential during a pandemic as it can reduce the likelihood that essential equipment, such as enteral feeding pumps, becomes unaccounted for. Dietetic emergency preparedness action plans require open communication across multiple disciplines to ensure adequate supply chain, accurate inventory, and strategies to manage issues such as equipment malfunction or misplaced supplies.

\section{Validation and Support from Leadership}

Clinical leaders should provide flexibility with RDN work schedules if a transition to a remote work environment is not feasible. The adjustment of policies and procedures so RDNs can still assess patients in a timely manner without feeling overwhelmed by the workload, along with the reduction or elimination of nonessential administrative tasks can also help a great deal. Leaders should regularly check in with RDN staff and proactively offer support and resources if a staff member is experiencing any mental health issues. Finally, it is recommended that clinical leaders provide support and assurance to staff should they require to be quarantined and promote inclusiveness by providing them with regular updates during their time under isolation.

\section{Inter-Institutional Sharing and Problem Solving}

Involve RDNs in the decision-making process regarding obstacles that will likely arise with the allocation of nutrition resources such as enteral feeding pumps as they may need to be prioritized during a pandemic. ${ }^{18}$ Nutrition management communication with other institutions should occur regularly as policies and procedures involving patient care will likely need adjustments. RDNs could also be represented in bioethics committees when issues involving nutrition arise such as lack of enteral formulas, feeding tubes and enteral feeding pumps. ${ }^{18}$ 


\section{RDN SKILLS, COMPETENCIES, AND SUPPORT}

\section{RDN Cross-Training and Nutrition Pandemic Resources}

Redeployment of a healthcare worker can be a source of stress, given they must work outside their specialty. ${ }^{19}$ It is important for RDNs to become more comfortable working with patient populations they normally do not engage with. RDN cross training and the development of clinical nutrition pandemic resources can help with decreasing stress levels and promoting a better work environment. RDNs will feel better prepared to handle an influx of patients and possible unit reassignment that can likely occur during a pandemic.

\section{Resilience and Coping Education}

Work performance may be the last thing to manifest as an issue as a direct result of an employee's stress level. ${ }^{20}$ Staff and clinical leaders should become familiar with the notable signs for potential mental health imbalances such as increased irritability or isolation in order to be able to obtain appropriate guidance and resources to help those in need. These techniques can be considered by RDNs early on when faced with a pandemic situation. ${ }^{20,21}$

- Be mindful of what is in your control \& what is not

- Keep in regular contact with family \& friends

- Make time for self-care (i.e. eating, staying hydrated, getting enough rest)

- Taking a break from social media \& the news

- Become familiar with resources that are offered at your institution (chaplain and/or counseling services, support groups)

\section{Mental Health Services}

The experience of a stressful or traumatic event, such as a pandemic, increases the risk for mental health issues. The term "burnout" is often associated with failure to handle stress and pressure at work, and the environment is often the reason that stress is able to take shape and put a strain on one's mental well-being. ${ }^{20}$ Validating the stressful situation and encouraging employees to be mindful, set boundaries, and to know their personal limitations can help a great deal. "A healthy work environment should be the standard, not the exception."20

\section{Resources}

\begin{tabular}{|l|l|}
\hline $\begin{array}{l}\text { National Suicide Prevention Hotline } \\
\text { 800-273-TALK (8255) } \\
\text { www.suicidepreventionlifeline.org }\end{array}$ & $\begin{array}{l}\text { Substance Abuse \& Mental Health Services Administration } \\
\text { 800-662-HELP (4357) } \\
\text { www.samhsa.gov }\end{array}$ \\
\hline $\begin{array}{l}\text { Crisis Text Line } \\
\text { Text "HOME" or "HELLO" to 741741 } \\
\text { www.crisistextline.org }\end{array}$ & $\begin{array}{l}\text { Disaster Distress Helpline } \\
\text { 800-985-5990 or }\end{array}$ \\
\hline
\end{tabular}

\section{REFERENCES}

1. Handu D, Moloney L, Rozga M, Cheng F. Malnutrition Care during the COVID-19 Pandemic: Considerations for Registered Dietitian Nutritionists Evidence Analysis Center. J Acad Nutr Diet. 2020.

2. Martindale R, Patel JJ, Taylor B, Arabi YM, Warren M, McClave SA. Nutrition Therapy in Critically III Patients with Coronavirus Disease (COVID-19). Journal of Parenteral and Enteral Nutrition. 2020.

3. Blake H, Bermingham F, Johnson G, Tabner A. Mitigating the Psychological Impact of COVID-19 on Healthcare Workers: A Digital Learning Package. International journal of environmental research and public health. 2020;17(9).

4. Bohlken J, Schömig F, Lemke MR, Pumberger M, Riedel-Heller SG. [COVID-19 Pandemic: Stress Experience of Healthcare Workers - A Short Current Review]. Psychiatr Prax. 2020;47(4):190-197.

5. Dutton RP, Grissom TE, Herbstreit F. COVID-19 and Trauma Care: Improvise, Adapt and Overcome! Anesth Analg. 2020.

6. Horesh D, Brown AD. Traumatic stress in the age of COVID-19: A call to close critical gaps and adapt to new realities. Psychol Trauma. 2020;12(4):331-335.

7. Walton M, Murray E, Christian MD. Mental health care for medical staff and affiliated healthcare workers during the COVID-19 pandemic. Eur Heart J Acute Cardiovasc Care. 2020;9(3):241-247.

8. Zaka A, Shamloo SE, Fiorente P, Tafuri A. COVID-19 pandemic as a watershed moment: A call for systematic psychological health care for frontline medical staff. J Health Psychol. 2020;25(7):883-887. 
9. Aamodt MG, Keller RJ, Crawford KJ, Kimbrough WW. A Critical-Incident Job Analysis of the University Housing Resident Assistant Position. Psychological Reports. 1981;49(3):983-986.

10. Morgeson FP, Dierdorff EC. Work analysis: From technique to theory. 2011.

11. Flanagan JC. The critical incident technique. Psychological bulletin. 1954;51(4):327.

12. Anderson L, Wilson S. Critical incident technique. 1997.

13. Luker KA, Austin L, Caress A, Hallett CE. The importance of 'knowing the patient': community nurses' constructions of quality in providing palliative care. Journal of Advanced Nursing. 2000;31(4):775-782.

14. Barberis N, Cernaro V, Costa S, et al. The relationship between coping, emotion regulation, and quality of life of patients on dialysis. The International Journal of Psychiatry in Medicine. 2017;52(2):111-123.

15. Conley CC, Bishop BT, Andersen BL. Emotions and emotion regulation in breast cancer survivorship. Paper presented at: Healthcare2016.

16. Dewey C, Hingle S, Goelz E, Linzer M. Supporting clinicians during the COVID-19 pandemic. In: American College of Physicians; 2020.

17. Baseman JG, Revere D, Painter I, Toyoji M, Thiede H, Duchin J. Public health communications and alert fatigue. BMC health services research. 2013;13(1):1-8.

18. Barrocas A, Schwartz DB, Hasse JM, Seres DS, Mueller CM. Ethical Framework for Nutrition Support Resource Allocation During Shortages: Lessons From COVID-19. Nutrition in Clinical Practice. 2020.

19. Akgün KM, Collett D, Feder SL, Shamas T, Schulman-Green D. Sustaining frontline ICU healthcare workers during the COVID-19 pandemic and beyond. Heart \& Lung: The Journal of Cardiopulmonary and Acute Care. 2020;49(4):346347.

20. Braddock J. Mental Health and the Registered Dietitian Nutritionist. Journal of the Academy of Nutrition and Dietetics. 2019;119(12):2109-2112.

21. Russell M. As Caring Professionals, Remember to Care for Ourselves. Journal of the Academy of Nutrition and Dietetics. 2019;119(5):719. 